\title{
EchoGéo
}

41 | 2017

Un état des lieux du Brésil en 2017

\section{São Paulo, du centre à la périphérie, les contrastes d'une mégapole brésilienne}

\section{Hervé Théry}

\section{(2) OpenEdition \\ 1 Journals}

\section{Electronic version}

URL: https://journals.openedition.org/echogeo/15088

DOI: $10.4000 /$ echogeo. 15088

ISSN: 1963-1197

\section{Publisher}

Pôle de recherche pour l'organisation et la diffusion de l'information géographique (CNRS UMR 8586)

\section{Electronic reference}

Hervé Théry, "São Paulo, du centre à la périphérie, les contrastes d'une mégapole brésilienne", EchoGéo [Online], 41 | 2017, Online since 28 September 2017, connection on 01 August 2021. URL: http:// journals.openedition.org/echogeo/15088 ; DOl: https://doi.org/10.4000/echogeo.15088

This text was automatically generated on 1 August 2021

EchoGéo est mis à disposition selon les termes de la licence Creative Commons Attribution - Pas d'Utilisation Commerciale - Pas de Modification 4.0 International (CC BY-NC-ND) 


\title{
São Paulo, du centre à la périphérie, les contrastes d'une mégapole brésilienne
}

\author{
Hervé Théry
}

1 La capitale du Brésil est Brasilia, mais São Paulo est - de loin - sa principale métropole, par sa population (une des dix plus peuplées à l'échelle mondiale), par son poids économique (c'est le plus gros pôle industriel de l'hémisphère sud) et par son rôle de polarisation de l'espace national. La petite mission fondée par les Jésuites en 1554 est devenue, au terme d'une croissance tardive mais rapide, la première agglomération du continent. Au moment de l'indépendance, en 1822, la ville comptait à peine 7000 habitants et elle avait été choisie comme siège d'une des deux premières Facultés de Droit du pays, en 1827, justement parce que c'était une petite ville calme, où les étudiants pourraient travailler en paix, comme à Cambridge ou à Heidelberg.

2 Une extraordinaire croissance a ensuite fait passer São Paulo de 31000 habitants en 1872 à 19 millions en 2017 (sa population ayant donc été multipliée par plus de 600 en 145 ans). Au terme d'une expansion anarchique et dévoreuse d'espace, elle couvre aujourd'hui plus de $8000 \mathrm{~km}^{2}$ ( 80 fois la superficie de la ville de Paris et près de trois fois celle de l'agglomération parisienne). 
Illustration 1 - La croissance de São Paulo

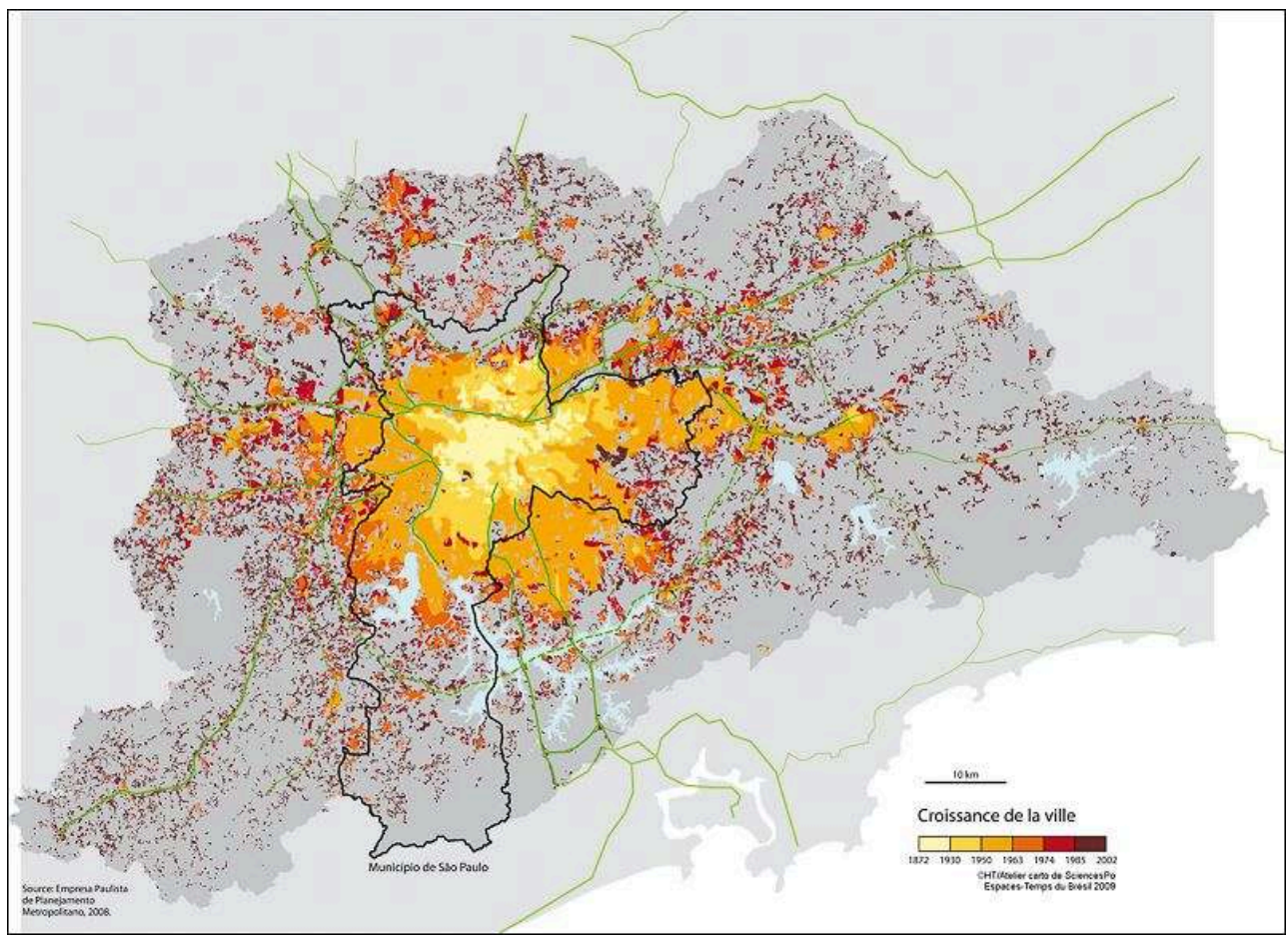

3 Cette agglomération géante est marquée par de très fortes disparités dans l'usage du sol, la qualité du bâti et les niveaux de revenus de ses populations, à l'image des contrastes qui caractérisent le Brésil dans son ensemble. Une très nette zonation sociale apparaît ainsi entre une zone centrale, parfois déjà dégradée, une zone sudouest où sont concentrés les ménages à revenus élevés, des quartiers intermédiaires et une énorme périphérie pauvre. Les photos qui suivent forment un transect du centre à la périphérie, pour rendre compte de ce gradient, et sont localisées su la carte de l'illustration 2. 


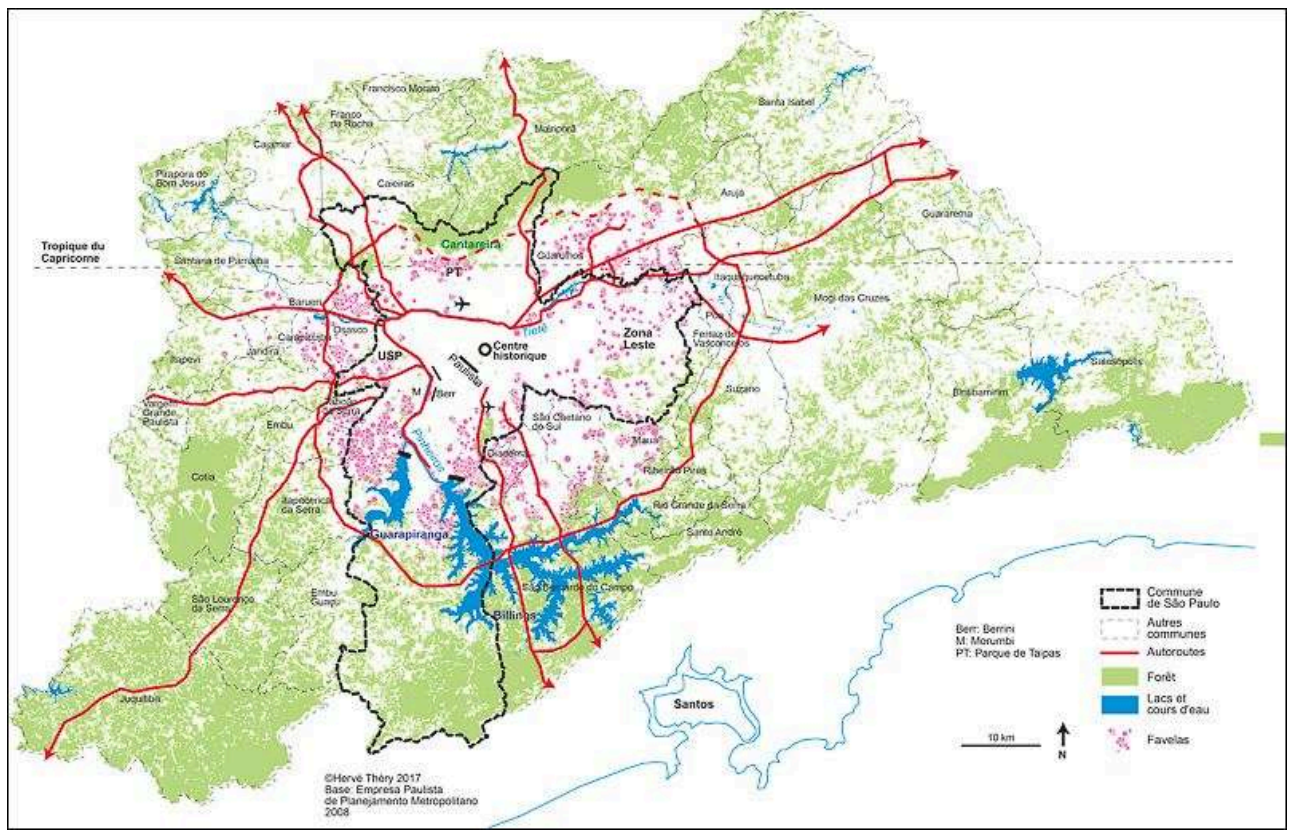

Illustration 3 - Le centre, l'axe de l'Avenida Paulista

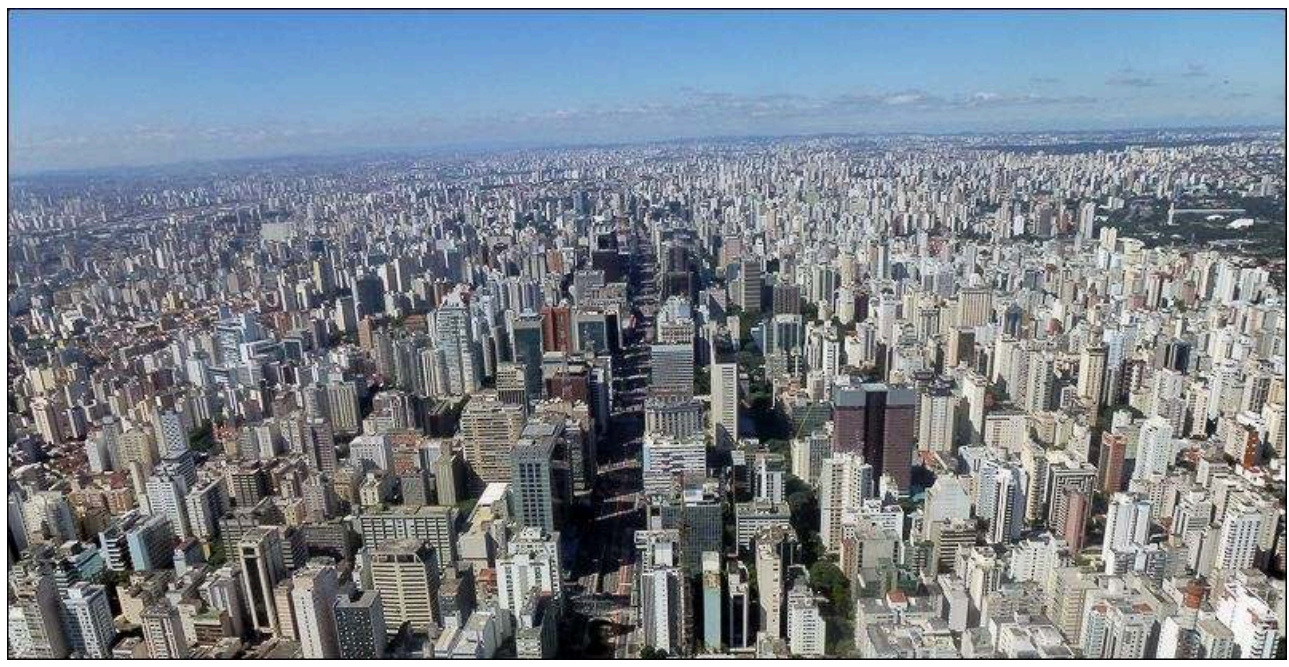

Auteur: H. Théry.

4 Le centre de la ville est un des paysages urbains les plus étonnants du pays, avec sa forêt de tours, la circulation automobile et les embouteillages qui le bloquent régulièrement. Un jour par semaine, la circulation y est interdite aux heures de pointe à un cinquième des véhicules, en fonction de leur numéro de plaque minéralogique (1 et 2 le lundi, 3 et 4 le mardi, etc.). Ce « centre étendu », compris entre les rios Tietê et Pinheiros, n'est plus qu'une petite partie de la ville et autour de lui, intercalées dans le bâti résidentiel, cohabitent des zones industrielles et commerciales, des favelas, des zones de loisirs et bon nombre de friches en attente de valorisation. 
Illustration 4 - Le collège des Jésuites où tout a commencé

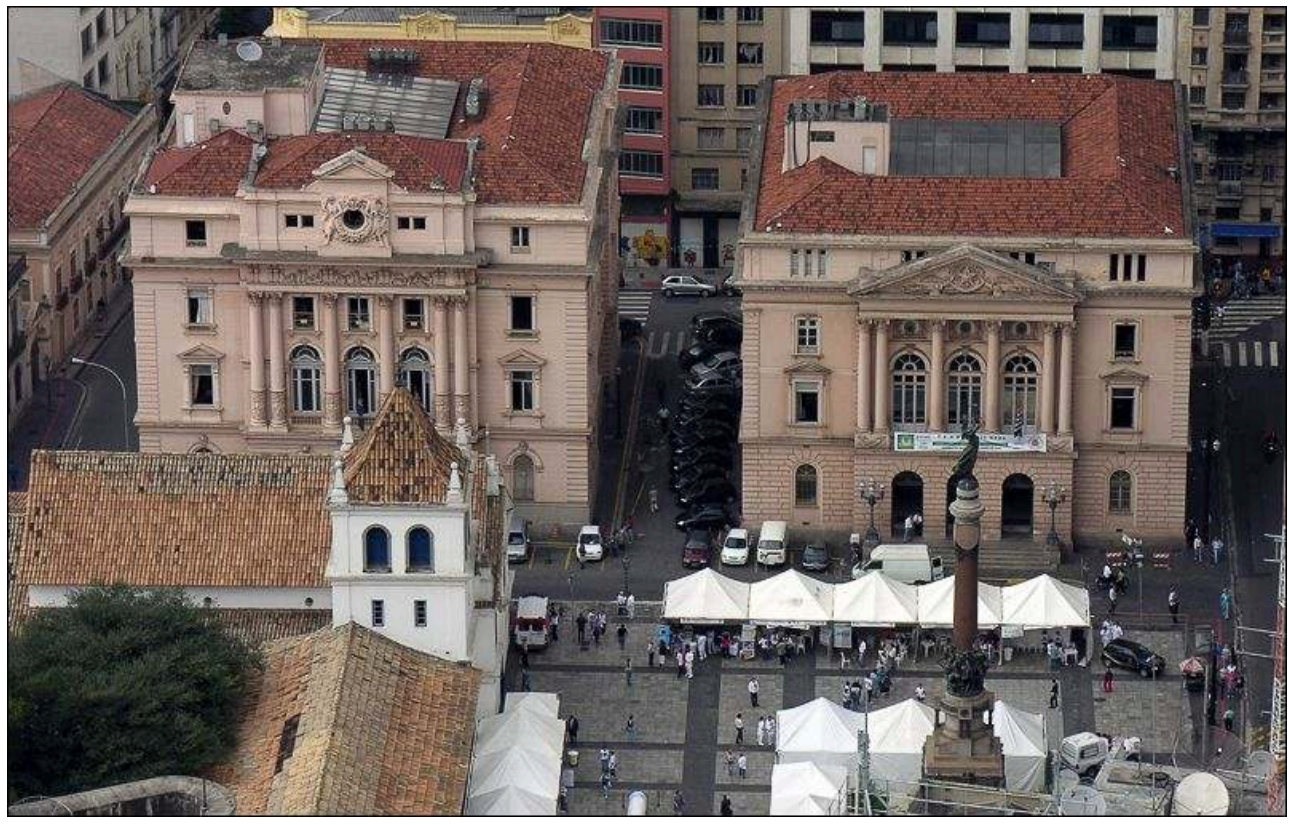

Auteur : H. Théry.

5 Le collège fondé par les Jésuites en 1554 a été le premier bâtiment construit à São Paulo, pour catéchiser les Indiens. Il a été implanté sur le plateau sommital de la Serra do Mar, alors que les Portugais avaient jusque-là leur base au bord de la mer, à São Vicente (aujourd'hui englobée dans l'agglomération de Santos (à $70 \mathrm{~km}$ de là). Sur le même site cohabitent aujourd'hui une église et ses dépendances, reconstruites en style portugais de multiples réformes du temps où le bâtiment était le siège du gouvernement de São Paulo. Au second plan deux bâtiments jumeaux, de style néoclassique, abritent des secretarias (ministères) de l'État de São Paulo. Ils témoignent de la volonté des élites paulistes, à la fin du XIX siècle, de manifester que leur modèle de développement n'était plus la péninsule Ibérique, mais l'Europe du Nord. 
Illustration 5 - Un palacete de l'Avenida Paulista et son successeur

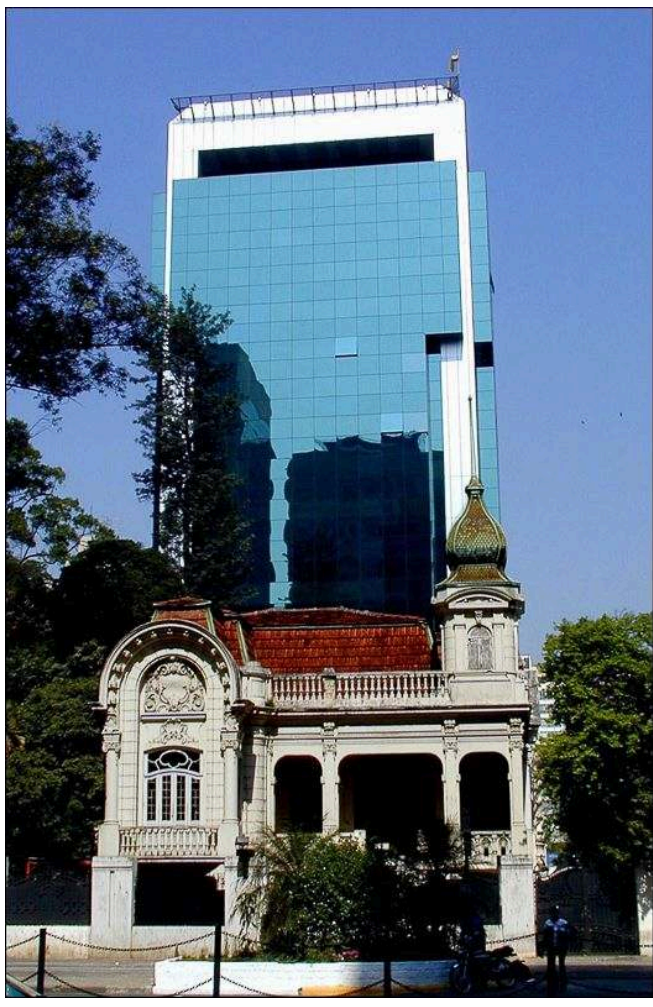

Auteur: H. Théry.

6 L'Avenida Paulista a été inaugurée en 1891. Contrairement aux Campos Elíseos et à Higienópolis, quartiers où vivaient les barons du café, l'avenue Paulista est devenue le lieu de prédilection des immigrants enrichis dans l'industrie naissante de São Paulo. Pour les nouveaux riches qui choisissaient de s'installer sur l'Avenida Paulista la meilleure façon de prouver leur réussite était de faire édifier des «palacetes " (que l'on peut traduire par " petit palais ») en rivalisant de luxe avec leurs voisins. Les artisans spécialisés capables de réaliser la décoration (stucs, vitraux, parquets, etc.) venaient pour la plupart d'Europe, principalement d'Italie et de France, ainsi que bien souvent les matériaux de construction et de décoration.

7 Il reste hélas bien peu de ces palacetes sur la Paulista, à part ceux où il a été possible de construire un immeuble moderne dans le jardin et de garder le bâtiment d'origine comme une entrée prestigieuse du nouveau bâtiment. Une grande partie des demeures de l'avenue étaient encore debout en juin 1982, quand une déclaration maladroite du président du conseil chargé de la préservation du patrimoine historique, selon laquelle il envisageait le classement de certaines de ces maisons, incita les propriétaires à les détruire dans la nuit, sans licence de démolition. 
Illustration 6 - Jardins suspendus devant l'Avenida Paulista

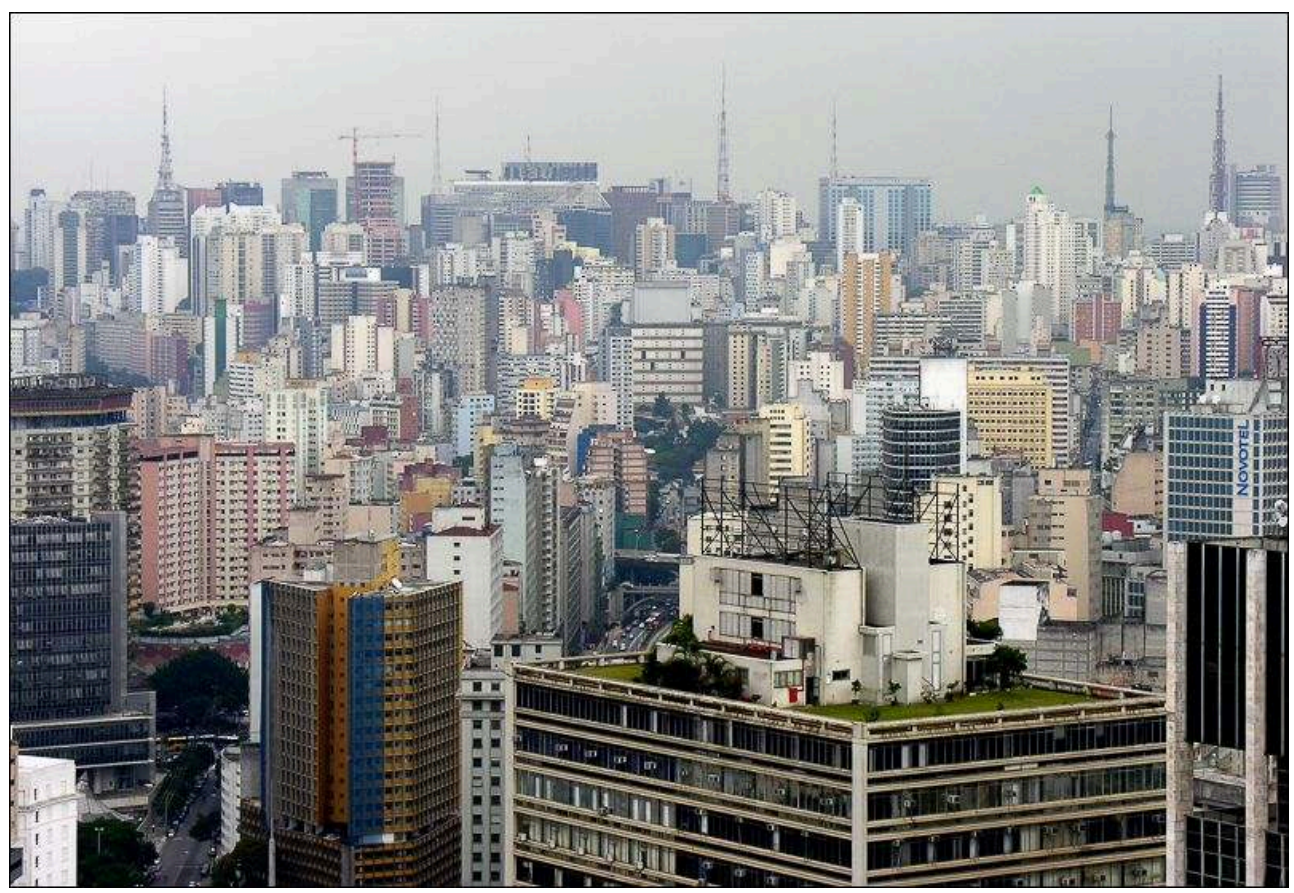

Auteur: H. Théry.

8 L'Avenida Paulista est aujourd'hui un quartier verticalisé. Elle a été, dans les années 1950-1970 le cœur économique et financier de la ville, avant que celui-ci ne se déplace vers des quartiers plus modernes encore, le dernier en date étant celui de l'avenue Berrini. L'avenue ayant été en partie délaissée par le monde des affaires, les propriétaires des immeubles et la mairie ont dû lui chercher de nouvelles fonctions et en ont trouvé avec le commerce et les loisirs. L'avenue est désormais piétonne plusieurs dimanches par mois et devient un lieu de promenade pour les habitants du quartier et pour d'autres qui viennent de plus loin pour y flâner. Certains privilégiés peuvent même s'y faire construire des villas au sommet d'immeubles naguère réservés aux bureaux. 


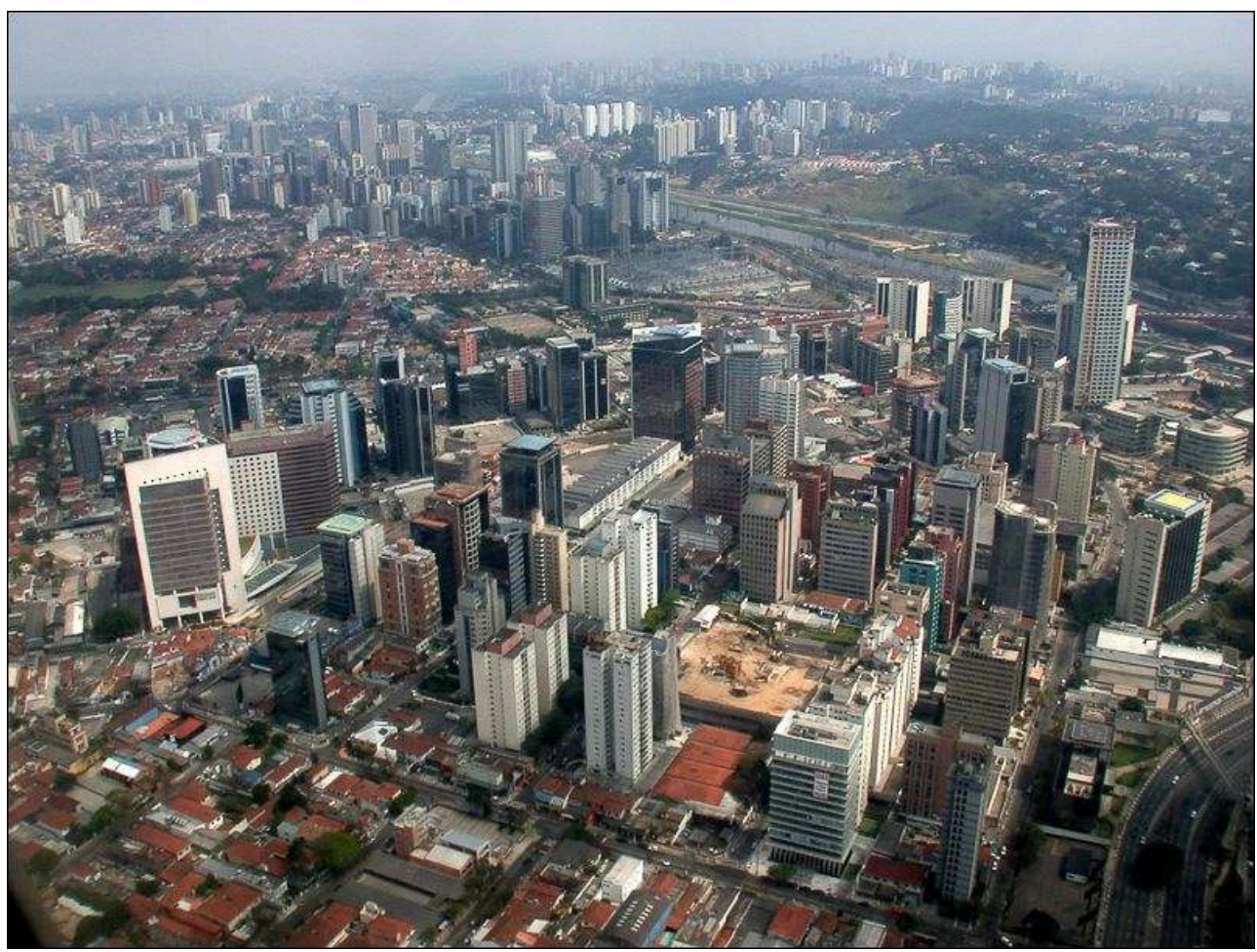

Auteur: H. Théry

9 Au premier plan le quartier des affaires de l'avenue Berrini, où de nombreux immeubles disposent d'un héliport à leur sommet (on en distingue 17 sur la photo), ce qui permet aux hommes d'affaires d'échapper aux embouteillages. La ville compte plus de 800 hélicoptères (la $2^{\mathrm{e}}$ flotte mondiale après New York) et un contrôle aérien spécial pour surveiller leurs évolutions et faire respecter leurs couloirs aériens.

En arrière-plan le Pinheiros, une des deux rivières qui délimitent le centre-ville. Le centre historique est en perte de vitesse, au profit du sud-ouest, où se sont développés successivement un deuxième centre d'affaires, au long de l'avenue Paulista, un troisième vers le rio Pinheiros, autour des nouveaux shopping centers et des centre d'affaires de l'avenue Faria Lima, puis un quatrième, autour de l'avenue Berrini, où les multinationales trouvent les «immeubles intelligents", câblés et contrôlés par ordinateur, qu'elles recherchent pour installer leurs sièges sociaux. 
Illustration 8 - Match de futebol au stade Morumbi

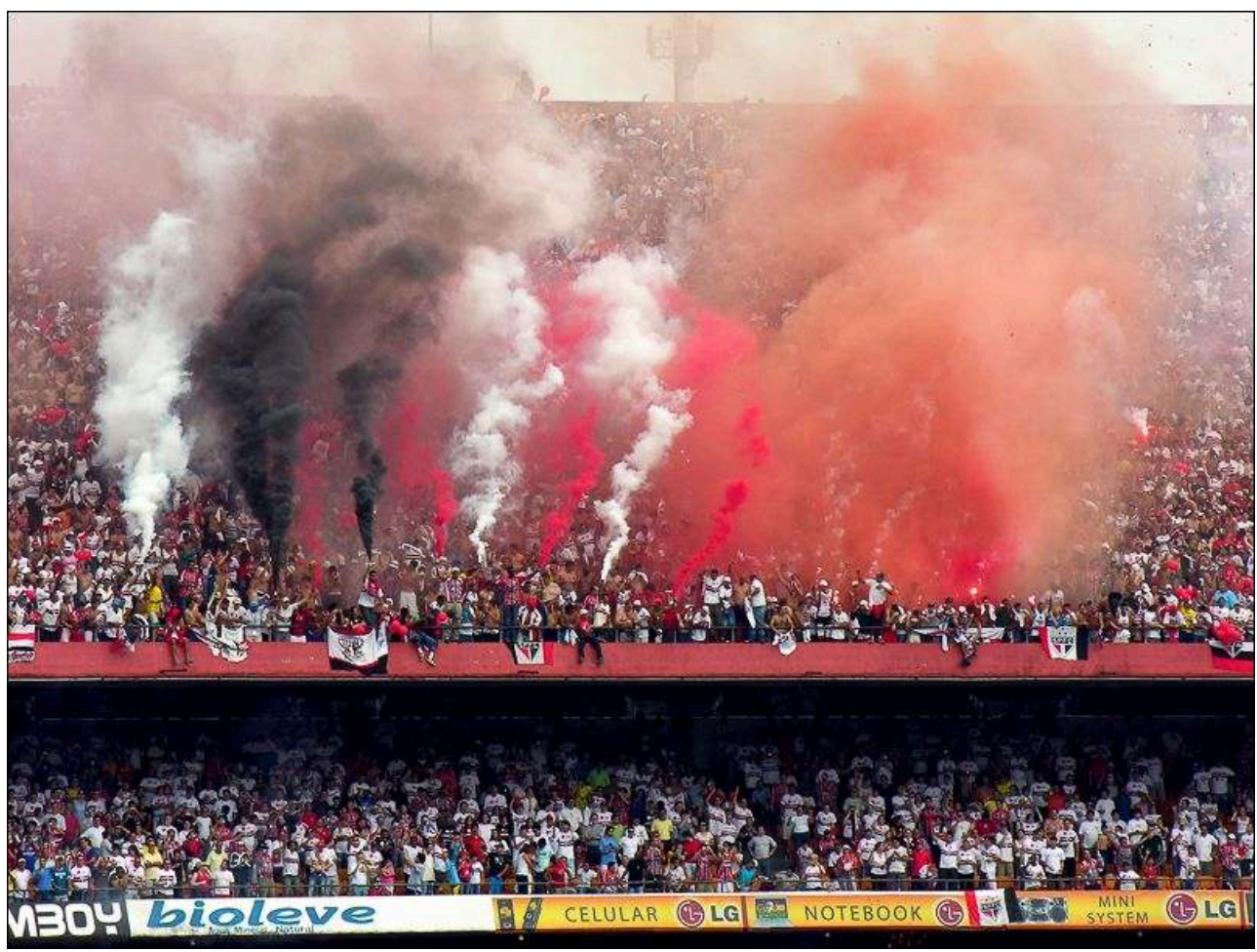

Auteur: H. Théry.

11 Le stade du Morumbi est le siège du São Paulo futebol clube (SPFC), un des grands clubs de São Paulo avec le Corintians, le Palmeiras qui sont, avec Santos, les grands rivaux des clubs de Rio de Janeiro. Une ville sans grande équipe peut-elle faire figure de métropole au Brésil ? Cette image, prise lors du match décisif qui lui permit de remporter le championnat national en 2006, montre l'enthousiasme de ses supporters (torcedores en portugais). Il lui manque toutefois le son, cris de la foule, sirènes et orchestres de percussions. 
Illustration 9 - Le parc Villalobos et le campus de l'USP (Universidade de São Paulo)

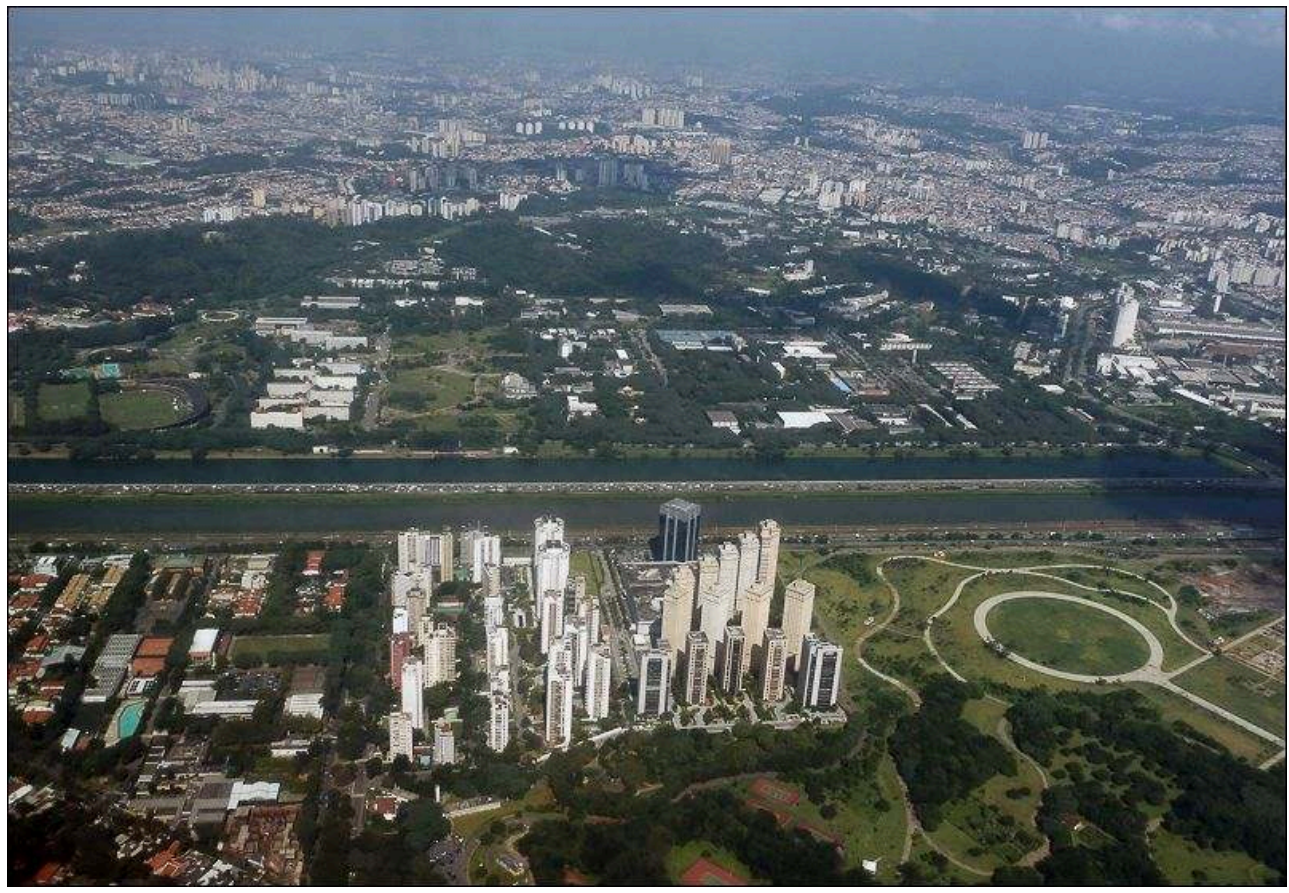

Auteur: H. Théry.

12 Au premier plan le parc Villalobos et le quartier d'immeubles construits autour du shopping center associé au parc. Au milieu de l'image la rivière Pinheiros, doublée par le bassin d'aviron construit pour les jeux panaméricains de 1963 sur le campus de l'Université de São Paulo, l'USP. Celle-ci occupe toute la zone boisée qui s'étend au-delà de la rivière, ses 58000 étudiants, 6000 professeurs et 15500 membres du personnel technique et administratif occupant un campus de près de 800 ha. Son rayonnement international est un élément essentiel d'une ville mondialisée. En août 2017 elle a été classée premières des universités latino-américaines et $151^{\mathrm{e}}$ mondiale par le classement Academic Ranking of World Universities (dit "de Shanghai »). Il est à noter qu'elle est financée l'État de São Paulo, et non par l'État fédéral, et que les études - après avoir passé avec succès le concours d'entrée - y sont gratuites; ainsi que l'accueil en cité universitaire. 
Illustration 10 - La favela Parque de Taipas et le Pico do Jaraguá

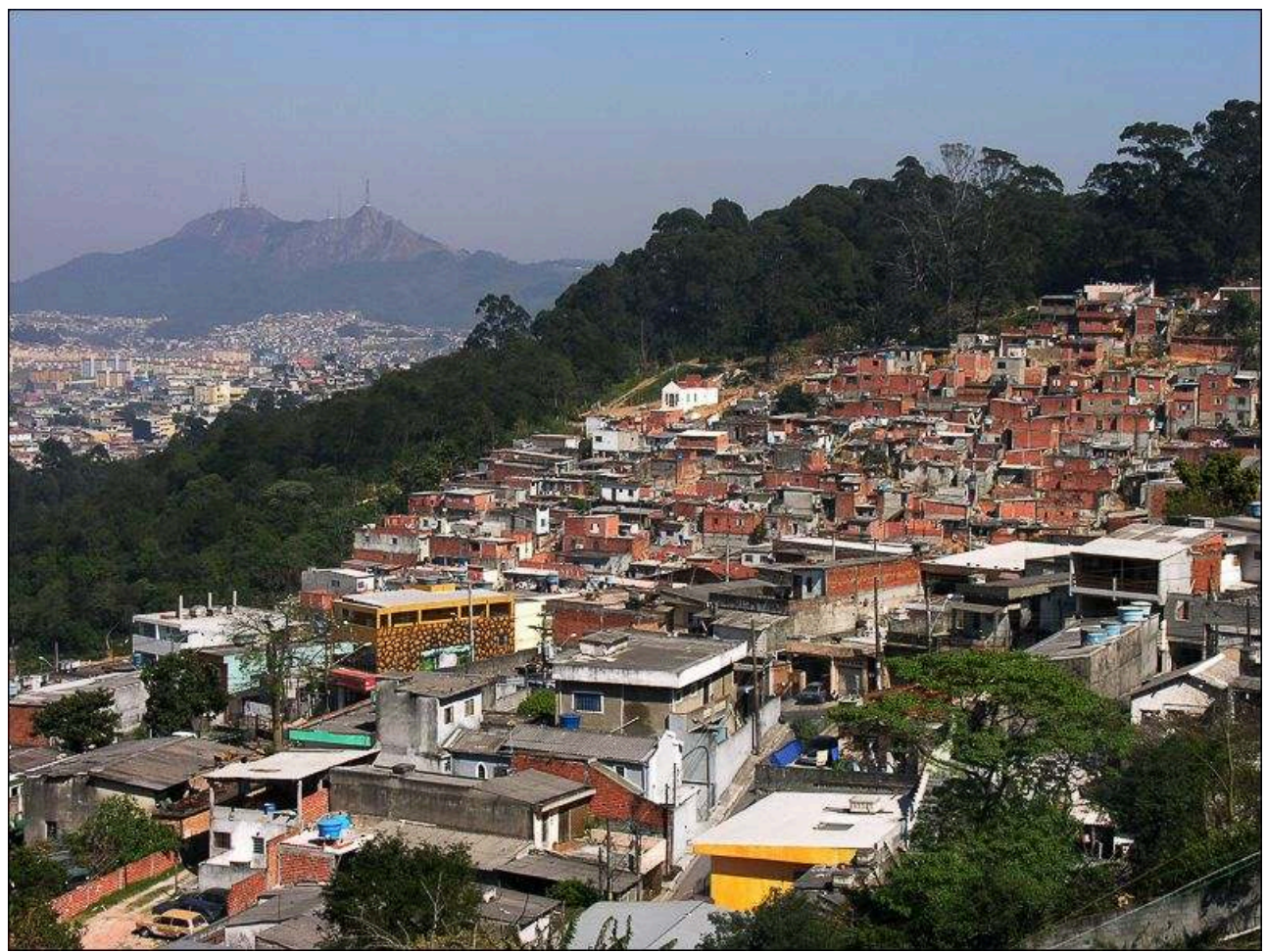

Auteur : H. Théry.

13 À l'autre extrémité de l'échelle sociale se situent les favelas où vivent les plus pauvres. São Paulo s'est longtemps vanté d'en avoir moins que Rio de Janeiro, ce n'est plus le cas aujourd'hui. C'est aussi dans la ville la plus peuplée du pays, que le plus grand nombre de familles vivent dans ces quartiers précaires et insalubres. La favela du Parque de Taipas (illustration 10) avance sur la forêt et des pentes en principe inconstructibles, devant le point culminant de la ville, le Pico do Jaraguá. 
Illustration 11 - Périphéries en auto-construction

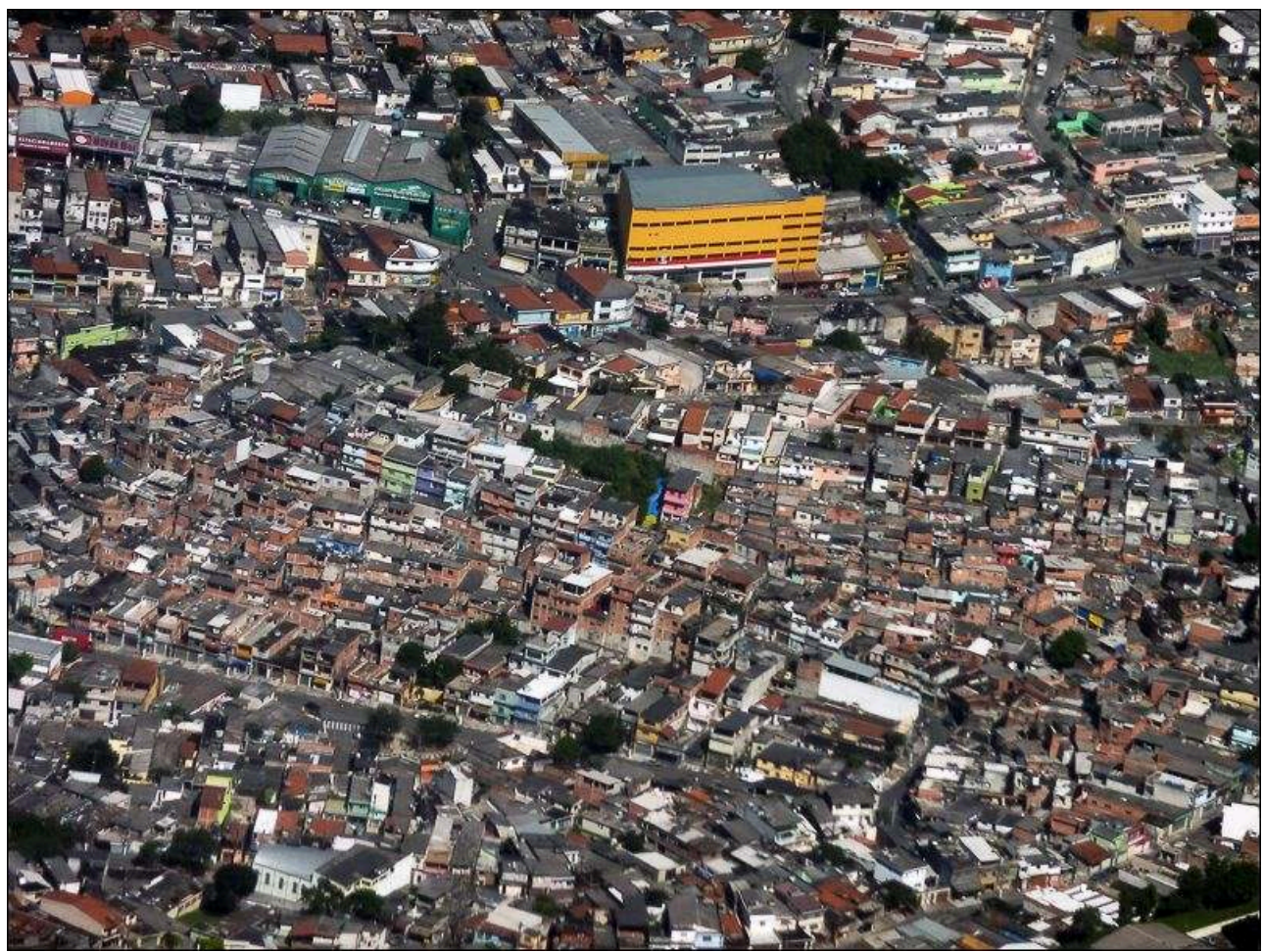

Auteur: H. Théry

14 La plus grande partie de la ville est formée par d'immenses périphéries où se sont entassés les millions de migrants qui rejoignaient la ville dans les années de croissance rapide (1940-1970). Mal bâties, sans aucune planification urbaine, elles forment la majorité du tissu urbain et posent d'insolubles problèmes de gestion urbaine aux autorités censées les développer.

Les habitants y ont construit eux-mêmes leurs maisons, souvent par étapes successives, en montant un mur ou un étage supplémentaire quand ils avaient quelques rentrées d'argent, sur des terrains censés être inconstructibles. Le résultat est un entassement serré de maisons très proches les unes des autres, sans rue bien tracée (et donc sans accès pour les services de sécurité), sans espaces verts ni espaces de loisirs. Les circulations se font par les rues principales, en bordure du quartier, alignements d'établissements commerciaux ou industriels hétéroclites, sans aucun souci de zonage.

Ces lotissements ne respectent pas les spécifications légales en matière de taille minimum, d'infrastructures et d'équipements, mais ils se font au vu et au su de tous, et sont périodiquement légalisés par des amnisties. Dans les quartiers les plus périphériques, moins d'un tiers des domiciles disposent d'eau courante, moins de $10 \%$ sont reliés au réseau d'égouts. 


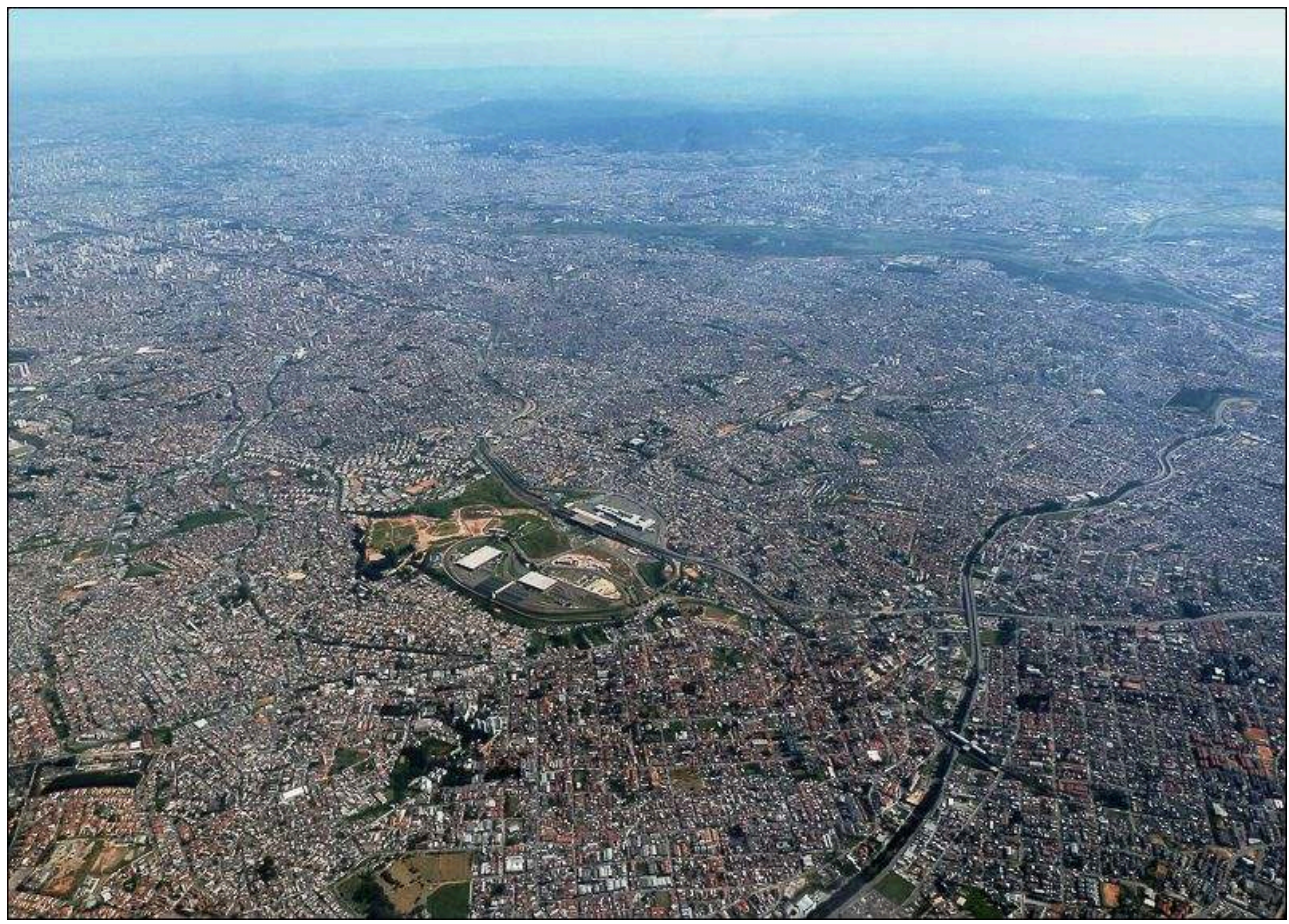

Auteur: H. Théry

17 La Zona Leste (zone Est), la plus grande de ces périphéries, compte plus de quatre millions et demi d'habitants. Elle est constituée essentiellement de ces quartiers dits d'auto-construction, sans planification ni structuration urbaine autour de grands axes, et présente un déficit en équipements publics et infrastructures de transports. C'est elle qui a en grande partie recueilli l'afflux des migrants, cause principale de la croissance démographique de la ville. Au milieu de l'image le chantier du stade ItaqueraCorintians, édifié pour la Coupe du monde de football de 2014. Construit sur la zone de manœuvre du terminal de métro (qui avait gardé des espaces libres) il est utilisé pour restructurer le quartier, à la manière du Stade de France de Saint Denis. Au fond le parc écologique du Tietê, aménagé sur les zones inondables de la rivière, le seul grand espace vert de la Zona Leste, au bord duquel a été installé le second campus de l'USP.

\section{Pour aller plus loin}

- «São Paulo, capitale du Brésil?», Géoconfluences, http://geoconfluences.ens-lyon.fr/ informations-scientifiques/dossiers-thematiques/de-villes-en-metropoles/corpus-

documentaire/portrait-sao-paulo-1-capitale

- «São Paulo, contrastes, problèmes, défis», Géoconfluences, http://geoconfluences.enslyon.fr/informations-scientifiques/dossiers-thematiques/de-villes-en-metropoles/corpusdocumentaire/portrait-sao-paulo-2-contrastes

\section{D'autres images}

Billet dans Braises (http://braises.hypotheses.org/) :

- Pluies tropicales sur São Paulo : http ://braises.hypotheses.org/86

- Appartements de luxe à São Paulo : http://braises.hypotheses.org/171

- Périphéries de São Paulo : http://braises hypotheses org/190

- Un « viaduc du thé » dans la capitale du café : http://braises hypotheses org/513

- Les embarras de São Paulo : http://braises.hypotheses.org/558 
- São Paulo vue du ciel : http://braises.hypotheses.org/1003

- Hiver à São Paulo : http://braises.hypotheses.org/1051

- Le Tietê, le fleuve de São Paulo : https://braises.hypotheses.org/1061

- La conquête de l'État de São Paulo vu par des cartes anciennes (1837-1932): https:// braises.hypotheses.org/1189

- Le Rodoanel, rocade autoroutière de São Paulo : https://braises.hypotheses.org/1282

Albums sur Flickr (https://www.flickr.com/) :

- São Paulo (20 photos) : https://flic.kr/s/aHsjt2VQtj

- Périphéries de São Paulo (8 photos) : https://flic.kr/s/aHsjwxBAGx

- São Paulo 1-5-16 (20 photos) : https://flic.kr/s/aHskvSG98D

\section{AUTHOR}

\section{HERVÉ THÉRY}

Hervé Théry, herve.thery@usp.br, est Directeur de recherche émérite au CNRS-Creda, Professeur à l'Universidade de São Paulo- USP (PPGH/FFLCH et GPP/EACH). 